\title{
Inducible deletion of CDK4 and CDK6 - deciphering CDK4/6 inhibitor effects in the hematopoietic system
}

Haematologica 2021

Volume 106(10):2624-2632

\section{Correspondence:}

VERONIKA SEXL

veronika.sex|@vetmeduni.ac.at

Received: April 22, 2020.

Accepted: August 21, 2020.

Pre-published: August 27,2020.

https://doi.org/10.3324/haematol.2020.256313

(C)2021 Ferrata Storti Foundation

Material published in Haematologica is covered by copyright. All rights are reserved to the Ferrata Storti Foundation. Use of published material is allowed under the following terms and conditions:

https://creativecommons.org/licenses/by-nc/4.0/legalcode. Copies of published material are allowed for personal or internal use. Sharing published material for non-commercial purposes is subject to the following conditions:

https://creativecommons.org/licenses/by-nc/4.0/legalcode, sect. 3. Reproducing and sharing published material for commercial purposes is not allowed without permission in writing from the publisher.

\section{Barbara Maurer,* Tania Brandstoetter,* Sebastian Kollmann, Veronika SexI," and Michaela Prchal-Murphy"}

Institute of Pharmacology and Toxicology, University of Veterinary Medicine, Vienna, Austria

${ }^{*} B M$ and $T B$ contributed equally as co-first authors.

"VS and MP-M contributed equally as co-senior authors.

\section{ABSTRACT}

yclin-dependent kinases 4 and 6 (CDK4/6) inhibitors are consid-
ered a breakthrough in cancer therapy. Currently approved for
breast cancer treatment, CDK4/6 inhibitors are extensively tested in other cancer subtypes. Frequently observed side effects include hematological abnormalities such as reduced numbers of neutrophils, erythroid cells and platelets that are associated with anemia, bleeding and a higher risk of infections. In order to understand whether the adverse effects within the hematopoietic system are related to CDK4 or CDK6 we generated transgenic mice that lack either CDK4 or CDK6 in adult hematopoiesis. Anemia and perturbed erythroid differentiation are associated with the absence of CDK6 but did not manifest in CDK4deficient mice. Total CDK6 knockout mice accumulate the most dormant fraction of hematopoietic stem cells due to an impaired exit of the quiescent state. We recapitulated this finding by deleting CDK6 in adult hematopoiesis. In addition, unlike total CDK6 knockout, all stem cell fractions were affected and increased in numbers. The deletion of CDK6 was also accompanied by neutropenia which is frequently seen in patients receiving CDK4/6 inhibitors. This was not the case in the absence of CDK4; CDK4 deficiency resulted in elevated numbers of myeloid progenitors without translating into numeric changes of differentiated myeloid cells. By using $C d k 4^{\mathrm{t} / \mathrm{II}}$ and $C d k b^{\mathrm{tl} / \mathrm{l}}$ mice we assign side effects of CDK4/6 inhibitors predominantly to the absence of CDK6. These mice represent a novel and powerful tool that will enable to study the distinct functions of CDK4 and CDK6 in a tissue-dependent manner.

\section{Introduction}

Cyclin-dependent kinases (CDK) 4 and 6 are best known for their roles in the mammalian cell cycle. Both are ubiquitously expressed, share $71 \%$ sequence homology and form complexes by binding to D-type cyclins (D1, D2 and D3). ${ }^{1}$ Cyclin D-CDK complexes phosphorylate the gatekeeper retinoblastoma (RB) and the related pocket proteins $\mathrm{p} 107$ and p130. In the hyper-phosphorylated state, RB proteins dissociate from $\mathrm{E} 2 \mathrm{~F}$ transcription factors which subsequently are freed to initiate gene transcription allowing the transition of the cell cycle from G1 to $\mathrm{S}$ phase. ${ }^{2,3}$ The CDK4/6-cyclin D-RB-E2F pathway is one of the most frequently dysregulated pathways in cancer; about $40 \%$ of human tumors display alterations in these proteins. ${ }^{3,4}$

In murine models, the combined loss of CDK4 and CDK6 is lethal in late embryonic or early postnatal development, which was considered to be a consequence of severe anemia. ${ }^{5} \mathrm{CDK} 4$ or CDK6 deficiency alone is mostly tolerated in the hematopoietic system implying many overlapping or compensatory functions of both proteins: CDK6 knockout mice are viable, fertile and show hematopoietic 
alterations with small spleens due to impaired erythroid and megakaryocytic cell development, ${ }^{5}$ and a lower red blood cell (RBC) number accompanied by increased erythrocyte size. ${ }^{6}$ In addition CDK6 is required for normal Tcell development in the thymus ${ }^{5,7}$ and for myeloid differentiation., ${ }^{8,9}$ The International Mouse Phenotyping Consortium identified CDK4 knockout mice as "subviable" - less homozygous knockout mice than expected were born - with smaller embryo size, hypoplasia of various organs and infertility, pinpointing to incomplete compensation of CDK4 functions by other proteins..$^{10,11}$ Another CDK4-deficient mouse model is also smaller in size, prone to develop diabetes and sterility $y^{12,13}$ and, besides an increase in erythrocyte size, lacks any abnormality in adult hematopoiesis even in the combined absence of CDK2 ${ }^{14,15}$ Similar to other paralogues, ${ }^{10} \mathrm{CDK} 4$ is considered to be able to compensate some, but not all of CDK6's functions and vice versa - partly reflected in the described phenotypes of the total knockout models. These overlapping roles or additional compensatory effects cannot be studied in complete knockout mice due to adaption for the total protein loss starting in embryogenesis.

CDK6, but not CDK4, has recently been assigned an additional function as a transcriptional regulator: as such CDK6 modulates p53 and nuclear factor $\kappa B$ (NFкB) responses, both important under oncogenic stress. ${ }^{16-19}$ In addition, activation of hematopoietic stem cells (HSC) depends on CDK6's kinase-independent role; CDK6 is required to suppress the quiescence inducer Egr1..$^{20}$ In Bcell lymphoid leukemia, CDK6 is part of a transcriptional complex that induces the pro-angiogenic factor VEGF-A in a kinase-independent manner. ${ }^{21}$ CDK6 acts - at least partly kinase-independent - oncogenic in JAK2 ${ }^{\mathrm{V} 617 \mathrm{P}}$-driven myeloproliferative neoplasms by promoting NFKB signaling, proinflammatory cytokine production and inhibiting apoptosis. ${ }^{18}$ In FLT3-ITD ${ }^{+}$myeloid leukemia, CDK6 was reported to act as a transcriptional regulator in a kinasedependent manner and is required to induce the leukemogenic drivers FLT3 and PIM1.22

CDK4 and CDK6 dual inhibitors, which block the kinase activity by preventing ATP from binding to the kinase pocket, have successfully entered the clinics. Palbociclib (Ibrance ${ }^{\circledR}$ by Pfizer), ribociclib (Kisqali ${ }^{\oplus}$ by Novartis) and abemaciclib (Verzenios ${ }^{\circledast}$ by Lily) have been approved for HER2-negative, locally advanced or metastatic breast cancer ${ }^{23}$ and are currently undergoing clinical trials to test their efficacy in solid tumors and leukemia. ${ }^{24}$ Recently, CDK6 expression was identified to be a conserved and direct target of diverse NUP98-fusions driving aggressive forms of acute myeloid leukemia, which were demonstrated to be hypersensitive to CDK4/6 inhibition. ${ }^{25}$ The huge clinical success of palbociclib is not only based on its role in interfering with the cell cycle, but also on its capacity to induce a senescence-like phenotype in breast cancer cells and to enhance cancer cell immunogenicity ${ }^{26}$ and T-cell activation. ${ }^{27}$ Side effects of CDK4/6 inhibitor treatment affect mainly the hematopoietic system and include neutropenia, lymphopenia, anemia and thrombocytopenia. As a consequence the patients display an increased rate of infections, especially of the upper respiratory tract. . $^{2-30}$

As several proto-oncogenic effects of CDK6 are kinaseindependent, CDK4/6 inhibitors might target only parts of CDK6's functions. Specific protein degraders hijacking the cell's proteasomal degradation machinery called PROTACs represent novel alternatives to kinase inhibitors and have been reported to specifically target CDK6. ${ }^{31-33}$ These compounds circumvent the possible upregulation of CDK6 expression in CDK4/6 inhibitortreated patients leading to therapy resistance ${ }^{34,35}$ and target the kinase-independent regulatory roles of CDK6.

We here describe the generation of Mx1-Cre Cdkb and $\mathrm{Mx} 1-\mathrm{Cre} C d k^{\mathrm{f} / \mathrm{fl}}$ mice and their phenotype upon polyinosinic-polycytidylic acid (poly(I:C)) treatment. In contrast to total CDK4 or CKD6 knockout mice, which can develop compensatory mechanisms starting in embryogenesis and hematopoietic development, we study direct consequences of CDK4 or CDK6 loss in adult mice with a focus on hematopoiesis. Our study links the hematopoietic side effects of CDK4/6 inhibitors as anemia and neutropenia predominantly to CDK6 inhibition. Loss of CDK6 results in an accumulation of early HSC and common lymphoid progenitors (CLP), while CDK4deficient mice acquire an expansion of committed myeloid progenitors.

We validated the novel $C d k 4^{\mathrm{A} / \mathrm{I}}$ and $C d k 6^{\mathrm{H} / \mathrm{I}}$ mouse models as powerful tools to decipher the roles of CDK4 and CDK6 in any cell type-specific or inducible manner.

\section{Methods}

\section{Mouse strains}

$\mathrm{Cdk} 4^{\mathrm{B} /+}$ and $\mathrm{Cdk6^{ \textrm {H } / + }}$ mice were obtained from the Canadian Mouse Mutant Repository. The mouse line C57BL/6N$\mathrm{Cdk} 4 \mathrm{tm} 1 \mathrm{c}(\mathrm{NCOM}) \mathrm{Mfgc} / \mathrm{Tcp}$ (referred to as $\mathrm{Cdk} 4^{\mathrm{f} / \mathrm{I}}$ ) was generated as part of the NorCOMM2 project with C57BL/6NCdk4tm1a(NCOM)Mfgc/Tcp made from NorCOMM embryonic stem (ES) cells ${ }^{36}$ at the Toronto Center for Phenogenomics, Canada. The mouse line C57BL/6NCdk6tm1c(EUCOMM)Wtsi/Tcp (referred to as $C d k 6^{\mathrm{t} / \mathrm{l}}$ ) was generated with C57BL/6N-Cdk6tm1a(EUCOMM)Wtsi/Tcp made from EUCOMM ES cells ${ }^{36}$ at the Toronto Center for Phenogenomics, Canada.

Mice carrying homozygous floxed alleles were crossed with $\mathrm{Mx} 1-\mathrm{Cre}$ mice ${ }^{37}$ to allow inducible deletion of Cdk4 or Cdk6 in the hematopoietic system, respectively. Eight-week-old male and female mice were injected intraperitoneally (i.p.) with $200 \mu \mathrm{g}$ poly(I:C) every 3 days (three times in total). Analysis was done 3 or 6 weeks post injection. All mice were bred and maintained under pathogen-free conditions at the Institute of Pharmacology and Toxicology, University of Veterinary Medicine, Vienna. All procedures were approved by the Institutional Ethics and Animal Welfare Committee and the national authority according to \$\$26ff. of the Animal Experiment Act, Tierversuchsgesetz 2012 TVG 2012 (GZ BMBWF-68.205/0174-V/3b/2018).

\section{Flow cytometry}

Single-cell suspensions were prepared from bone marrow (BM), spleen (including RBC lysis) and peripheral blood (no RBC lysis for erythroid development; including RBC lysis for lineage-restricted cells) and analyzed by flow cytometry using a FACS Canto II cell analyzer (BD Biosciences, Franklin Lakes, New Jersey, USA). For stem cell analysis, cells were stained as follows: lineage panel containing Pacific Blue-conjugated CD3, CD11b, Gr1, Ter119 and B220, Sca1-PeCy7, cKit-PerCPCy5.5, CD34-FITC, CD150-APC, CD135-APC-Cy7 and CD48-PE. For myeloid and lymphoid progenitors, the following antibodies were used: lineage panel containing Pacific Blue-conjugated CD3, CD11b, Gr1, Ter119 and 
B220, CD127-FITC, Sca1-PeCy7, cKit-PerCPCy5.5, CD16/32-PE and CD34-APC. For lineage-restricted cells, the following antibodies were used: Gr1-APC-eFlour780, CD3-Pacific Blue, CD19-APC, Ter119-PE and CD11b-PerCPCy5.5. Erythroid development was evaluated using Ter119-APC-eFluor780, CD71-Biotin and Streptavidin-PE-Cy7 antibodies (Ter119 ${ }^{\text {med }}$ CD71 ${ }^{\text {high }}$ (proerythroblasts; R1), Ter119 ${ }^{\text {high }} \mathrm{CD} 71^{\text {high }}$ (basophilic erythroblasts; R2), Ter119 ${ }^{\text {high }} \mathrm{CD} 1^{\text {med }}$ (late basophilic and polychromatophilic erythroblasts; R3) and Ter119 high $^{\text {he }}$ 7 $1^{\text {low }}$ (orthochromatophilic erythroblasts; R4). All antibodies were purchased from BD Biosciences, eBioscience (ThermoFisherScientific, Waltham, Massachusetts, USA) or BioLegend (San Diego, California, USA). Cell cycle analysis was performed in a two-step protocol by staining with antibodies directed against HSC surface markers (lineage panel containing APC-Cy7-conjugated CD3, CD11b, Gr1, Ter119 and B220, Sca1-PeCy7, cKit-PerCPCy5.5, CD150-APC and CD48PE) followed by intracellular staining with Ki-67 FITC and 4,6 diamidino-2-phenylindole (DAPI). For intracellular staining, cells were fixed in Cytofix/Cytoperm (BD Biosciences), washed and stained with Ki-67 FITC (BD Bioscience), and then co-stained with $0.1 \mathrm{mg} / 100 \mathrm{ml}$ DAPI in phosphate-buffered saline (PBS). Flow cytometry plots were evaluated by FACSDiva software version 6.1.2 or FlowJo v10.6.1 (both BD).

\section{Statistical analysis}

Statistical analysis was carried out using a one-way analysis of variance (ANOVA) with subsequent Bonferroni's or Tukey's multiple comparison test, Mann-Whitney U test or Kruskal-Wallis test with subsequent Dunn's test. Data are presented as mean values + standard error of the mean (SEM) and were analyzed by GraphPad Prism. Statistical significance is as follows: ${ }^{*} P<0.05,{ }^{* *} P<0.01$; ${ }^{* * *} P<0.001 ;{ }^{* * *} P<0.0001$.

\section{Results}

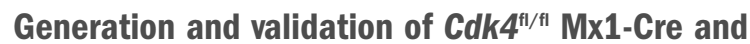

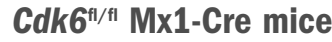

In order to explore distinct roles of CDK4 and CDK6 in adult hematopoiesis, we generated $\mathrm{Mx1}$-Cre $\mathrm{Cdk} \mathrm{f}^{\mathrm{H} / \mathrm{H}}$ and Mx1-Cre Cdk4 ${ }^{\text {t/l }}$ mice (Online Supplementary Figure S1A; Figure 1A). Heterozygous $\mathrm{Cdk}^{\mathrm{Al}+}$ or $\mathrm{Cdk}^{\mathrm{HI}+}$ mice on C57BL/6N background were obtained from the Canadian Mouse Mutant Repository and crossed to Mx1-Cre mice, which activate CRE recombinase upon poly(I:C) injection in hematopoietic cells. ${ }^{37}$ Eight-week-old Cdk4/1/1 Mx1-Cre,

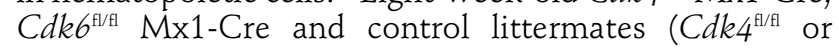
$\left.C d k 6^{\text {iflif }}\right)$ were injected three times with poly(I:C) and analyzed 3 or 6 weeks thereafter (Figure 1B). Polymerase chain reaction (PCR) analysis and western blot confirmed excellent deletion efficacy 3 weeks post poly(I:C) injection of Cdk4 or Cdk6 in spleen (Figure 1C and D) and BM (Online Supplementary Figure $S 1 B$ ) and 6 weeks after poly(I:C) injection (Online Supplementary Figure S1D) of Mx1-Cre expressing mice (hence called $C d k 4^{\Delta \Delta}$ or $\left.C d k b^{\Delta \Delta}\right)$. By trend, CDK4 expression is elevated upon Cdk6 deletion and vice versa (Figure 1E; Online Supplementary Figure S1C).

\section{CDK6 but not CDK4 influences erythroid development}

Patients treated with CDK4/6 inhibitors frequently develop anemia. ${ }^{28,38}$ In order to understand the contribution of $C D K 4$ or $C D K 6$ in this process, we studied erythroid development in $C d k 4^{\Delta \Delta}$ and $C d k 6^{\Delta / \Delta}$ mice and their littermate controls. $C d k 4^{\Delta \Delta}$ and $C d k b^{\Delta \Delta}$ mice displayed significantly reduced numbers of $\mathrm{RBC}$ in peripheral blood. The reduction was more pronounced in mice lacking Cdk6 (Figure 2A). Hematocrit and hemoglobin levels were only reduced in $C d k 6^{\Delta / \Delta}$ mice but not in $C d k 4^{\Delta / \Delta}$ animals (Online Supplementary Figure S2A and B). In complete $\mathrm{Cdkb}^{-1}$ mice, CDK6 is involved in the transition of mature $\mathrm{RBC}$ from the BM to the periphery. ${ }^{6}$ This is recapitulated in $C d k 6^{\Delta / \Delta}$ mice; they harbor elevated numbers of Ter119+ erythroid cells in the BM (Figure 2B) and show an altered maturation profile with a significant accumulation of cells in developmental stage R2 (basophilic erythroblasts) and R4 (orthochromatophilic erythroblasts). Erythroid development is unaffected in $C d k 4^{\Delta \Delta}$ mice (Figure $2 \mathrm{C}$ and D). The same effects were observed by trend when analyzing mice 6 weeks post Cdk4 or Cdk6 deletion (Online Supplementary Figure S2E). No changes were observed in the spleen size (Online Supplementary Figure S2C) or splenic numbers of erythroid cells (Online Supplementary Figure S2D) in $C d k 6^{\Delta \Delta}$ and $C d k 4^{\Delta \Delta}$ mice. These data led us to conclude that anemia observed upon CDK4/6 inhibitor treatment can be predominantly attributed to CDK6 blockade.

\section{Cdk6 $6^{\Delta / \Delta}$ mice accumulate hematopoietic stem/progenitor cells in the bone marrow}

CDK6 is required to exit quiescence in human HSC. ${ }^{39}$ In mice, CDK6 is crucial for HSC activation under stress while being dispensable under homeostatic conditions. $\mathrm{Cdkb}^{-1}$ mice display unaltered BM cellularity and LSK (Lin-Sca-1 ${ }^{+} \mathrm{c}-\mathrm{Kit}^{+}$) cell numbers. ${ }^{20}$ Based on CD150 and CD48 expression, LSK cells can be further subdivided in $\mathrm{CD} 150^{+} \mathrm{CD} 48$ Fraction A cells (containing the most dormant and long-term HSC), the more cycling CD $150^{+} \mathrm{CD} 48^{+}$Fraction B cells and CD150 CD48 Fraction C cells (myeloid- or lymphoid-prone) ${ }^{40} \mathrm{Cdk}^{-1}$ mice have comparable numbers of Fraction $\mathrm{A}, \mathrm{B}$ or $\mathrm{C}$ stem cells with a consistent increase of the most dormant HSC characterized by CD135CD34 within Fraction A cells. ${ }^{18,20}$ Upon deletion of CDK4 or CDK6 in adult BM we found that $C d k 4^{\Delta \Delta}$ mice reacted with a slight increase in BM cellularity while a subtle decrease was inflicted in $C d k 6^{\Delta \Delta}$ animals (Online Supplementary Figure S3A). Three weeks post Cdk4 or Cdk6 deletion, the percentage of LSK cells was significantly increased in $C d k 6^{\Delta \Delta} \mathrm{BM}$ but stayed unaltered in $C d k 4^{\Delta \Delta} \mathrm{BM}$ (Figure $3 \mathrm{~A}$ and $\mathrm{B}$ ). The change in LSK numbers in $C d k 6^{\Delta \Delta} \mathrm{BM}$ is attributed to the significant accumulation of long-term Fraction A stem cells, short-term Fraction B stem cells and multipotent progenitor (MPP) Fraction C cells (Online Supplementary Figure $S 3 B$ to D). Reminiscent of the findings in total $C d k b^{-1}$ mice, $C d k b^{\Delta \Delta}$ Fraction A stem cells were enriched with the most dormant HSC (CD150 CD $48^{+} \mathrm{CD} 135^{\circ} \mathrm{CD} 34^{-}$LSK), which was unaffected in $C d k 4^{\Delta / \Delta}$ BM (Figure 3C). Comparable results upon 6 weeks of poly(I:C) injection confirmed these findings (Online Supplementary Figure S3F).

In complete $\mathrm{Cdk}^{*}$ mice no significant changes in the cell cycle profile of Fractions A, B or C were detected under homeostatic conditions indicating a compensatory effect of CDK4 ${ }^{20}$ In $C d k 4^{\Delta \Delta}$, the proportion of Fraction A or $\mathrm{B}$ cells in each cell cycle phase was also unaltered compared to littermate control BM. In $C d k 6^{\Delta \Delta}$ mice a CDK4mediated compensation could not rescue the frequency of fraction $B$ cells in the $S / G_{2} / M$ phase which was significantly decreased (Figure 3D to E; Online Supplementary 
Figure S3E). These results underscore the dominance of CDK6 for the maintenance and proliferation of the stem cell pool.

\section{CDK4 and CDK6 effect the myeloid and lymphoid pro- genitor pool}

In complete $C d k b^{-1}$ mice, myeloid-primed progenitors (Lin c-Kit ${ }^{+} \mathrm{Sca}-1$; LKS) and their subpopulations including common myeloid progenitors (CMP), megakaryocyte/erythroid progenitors (MEP) and granulocyte/macrophage progenitors (GMP) are slightly increased. ${ }^{18}$ Three weeks upon deletion of CDK6 in adult hematopoiesis, the myeloid-primed progenitor pool remained unaltered in $C d k b^{\Delta \Delta} \mathrm{BM}$ as compared to littermate controls (Figure 4A; Online Supplementary Figure S4A and $B$ ). Myeloid-primed progenitors accumulated in $\mathrm{Cdk}^{\mathrm{s} / \Delta} \mathrm{BM}$ (Figure $4 \mathrm{~A}$ ) as did CMP (LKS CD34 $\left.{ }^{+} \mathrm{CD} 16 / 32^{10}\right)$, GMP (LKS CD34 $\left.{ }^{+} \mathrm{CD} 16 / 32^{\mathrm{hi}}\right)$ and MEP (LKSCD34CD16/32 $2^{\circ}$ ) (Online Supplementary Figure S4B). In contrast, 6 weeks after $C d k 4$ or $C d k 6$ deletion, the percentage of myeloid progenitors returns to an unaltered state in $C d k 4^{\Delta / \Delta}$ compared to controls, but increased in $C d k b^{\Delta / \Delta}$ (Online Supplementary Figure S4G) reflecting the $C d k 6^{-1-}$ phenotype. Independent of deletion span CLP (Lin IL-7R $\left.{ }^{+} \mathrm{c}-\mathrm{Kit}^{\mathrm{mid}} \mathrm{Sca}-1^{\mathrm{mid}}\right)$ are enriched only in $\mathrm{Cdk6^{ \Delta / \Delta }} \mathrm{BM}$ (Figure 4B; Online Supplementary Figure S4A and G) accompanied by elevated numbers of differentiated $\mathrm{CD}^{+}$ T cells (Figure 4D; Online Supplementary Figure $54 \mathrm{C}$ and $H$ ) and unchanged numbers of $\mathrm{CD}_{19}{ }^{+} \mathrm{B}$ cells (Online Supplementary Figure $S 4 C$ to $D$ ). Despite the lack of any
A
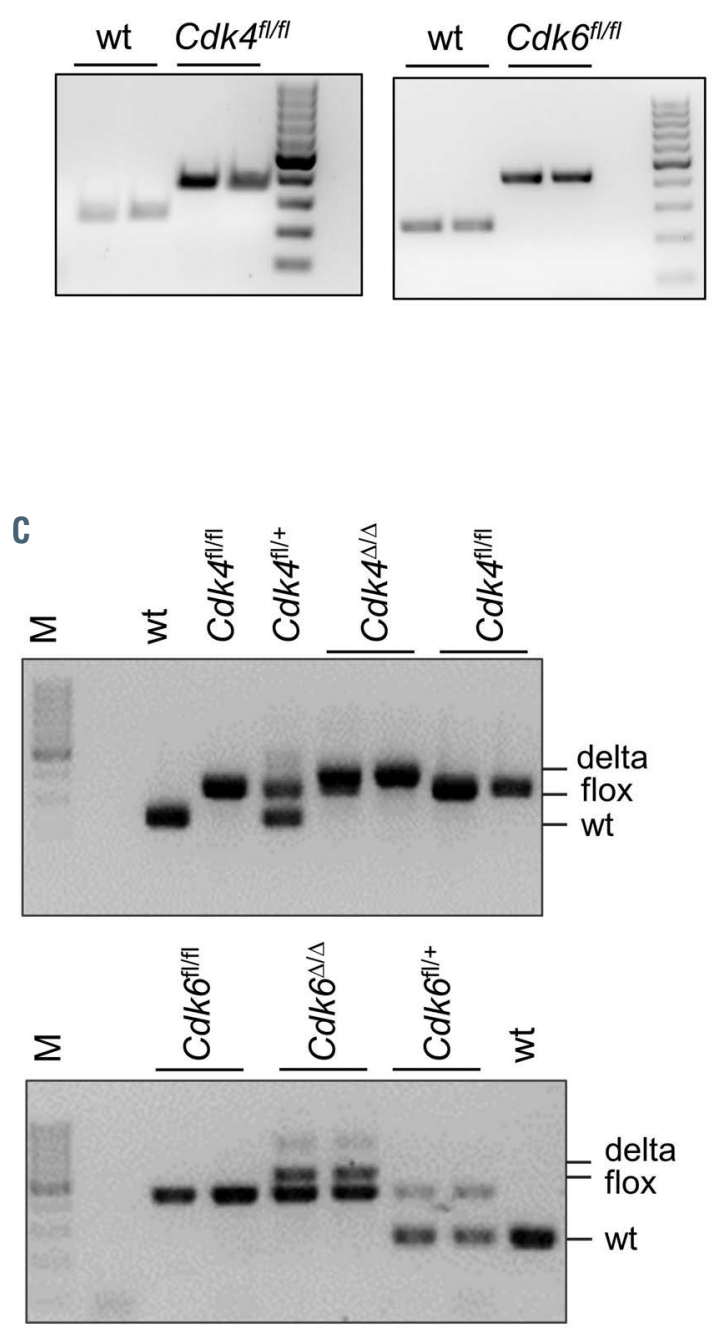

B

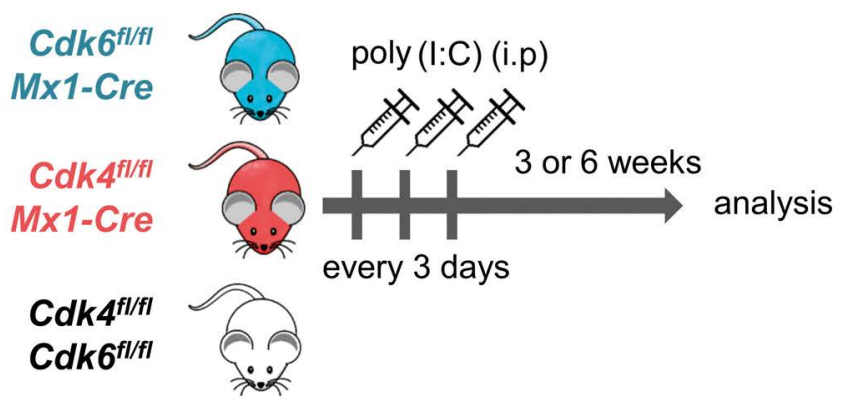

D

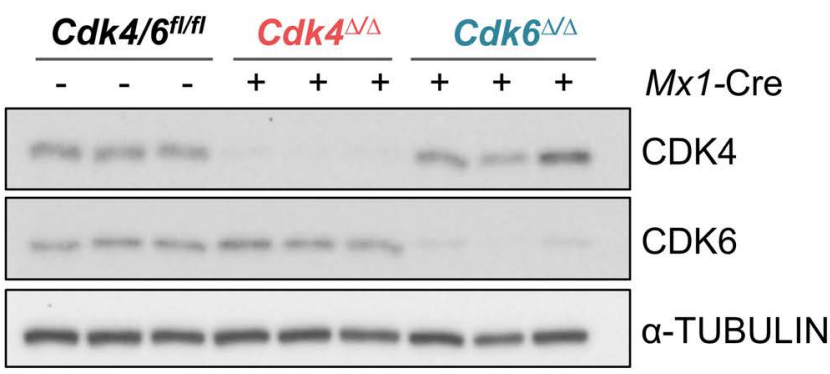

$\mathrm{E}$

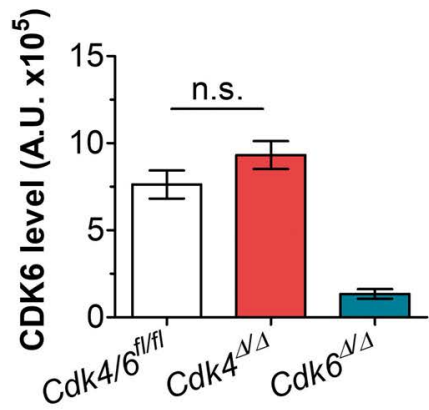

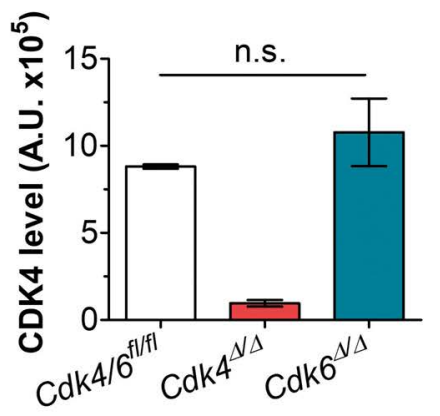

Figure 1. Reduced CDK4 or CDK6 expression in $C d k 4^{1 / / 1 / 1}$ or $C d k 6^{\mathrm{fl} / 1 / 1} \mathrm{Mx1-Cre} \mathrm{mice.} \mathrm{(A)} \mathrm{The} \mathrm{presence} \mathrm{of} \mathrm{loxP-flanked} \mathrm{Cdk4} \mathrm{(left)} \mathrm{or} \mathrm{Cdk6} \mathrm{(right)} \mathrm{were} \mathrm{confirmed} \mathrm{by} \mathrm{poly-}$ merase chain reaction (PCR) on genomic DNA of murine tissue, $100 \mathrm{bp}$ marker. (B) Treatment regime for Mx1-Cre mediated deletion of Cdk4 (Cdk4// ) or Cdk6 $\left(C d k 6^{\Delta / \Delta}\right)$ by polyinosinic-polycytidylic acid (poly(l:C)) injection (200 $\mu$ g, intraperitoneally, three times every 3 days). (C) Deletion PCR: confirmation of Cdk4 ${ }^{\Delta / \Delta}$ (top) or $C d k 6^{\Delta / \Delta}$ (bottom) DNA in splenocytes showing wild-type (wt), floxed and delta bands. Analysis was performed 3 weeks post final poly(l:C) injection. (D) Immunoblotting:

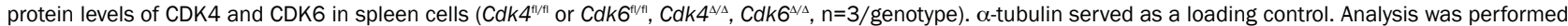
3 weeks post final poly(I:C) injection. A representative blot of at least three independent experiments is shown. (E) Quantification of immunoblot shown in (D), signal intensities were normalized to $\alpha$-tubulin levels (A.U. [arbitrary units]). Cdk4 $4^{\Delta / \Delta}$ or Cdk6 $6^{\Delta / \Delta}$ protein levels were compared to the respective controls by performing MannWhitney $\mathrm{U}$ tests. 
changes in the myeloid-primed progenitor pool in $C d k b^{\Delta \Delta}$ BM 3 weeks after Cdk6 deletion (Figure 4A; Online Supplementary Figure $S 4 A$ and $B$ ), the numbers of $\mathrm{CD} 11 \mathrm{~b}^{+} \mathrm{Gr} 1^{\text {hi }}$ differentiated granulocytes were drastically reduced in BM (Figure 4C; Online Supplementary Figure S4C) and blood (Online Supplementary Figure S4E) of $C d k b^{\Delta \Delta}$ mice, reminiscent to observations in $C d k b^{-/}$mice. This difference was less pronounced 6 weeks after $C d k 6$ deletion (Online Supplementary Figure S4H). The frequent pronounced drop in white blood cell (WBC) numbers that is observed in patients upon long-term CDK4/6 inhibitortreatment ${ }^{30}$ was not recapitulated in our experimental setting (Online Supplementary Figure S4F).

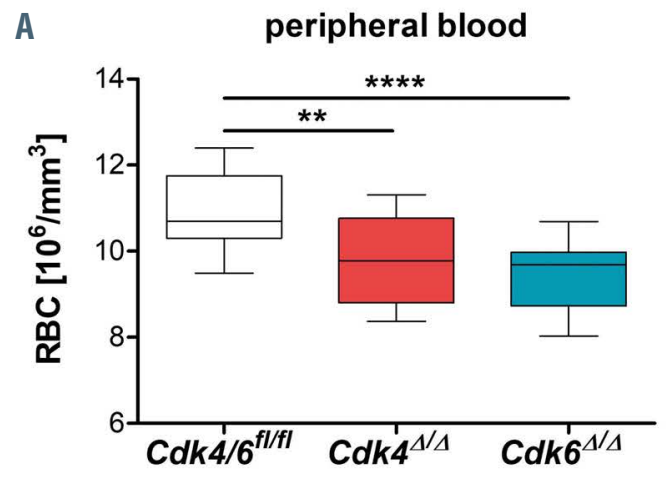

\section{Discussion}

The close homologues CDK4 and CDK6 play crucial roles in cell cycle progression and represent promising therapeutic targets in cancer. Inhibitors targeting both kinases have been approved for breast cancer treatment and are investigated in clinical trials for the treatment of additional solid and hematological malignancies. CDK4/6 inhibitor-associated side effects manifest primarily in the hematopoietic system and include anemia, neutropenia and lymphopenia. ${ }^{28,29,38}$ As attempts are ongoing to develop CDK inhibitors that will more specifically target individual $\mathrm{CDK}$ it is important to attribute side effects to either CDK4 or CDK6. We analyzed novel mouse models
B

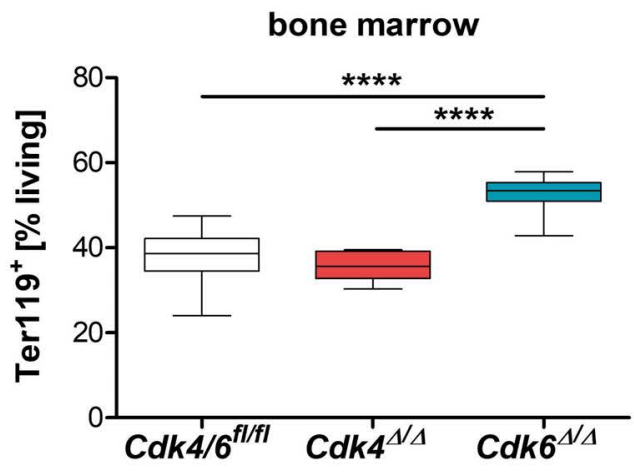

C

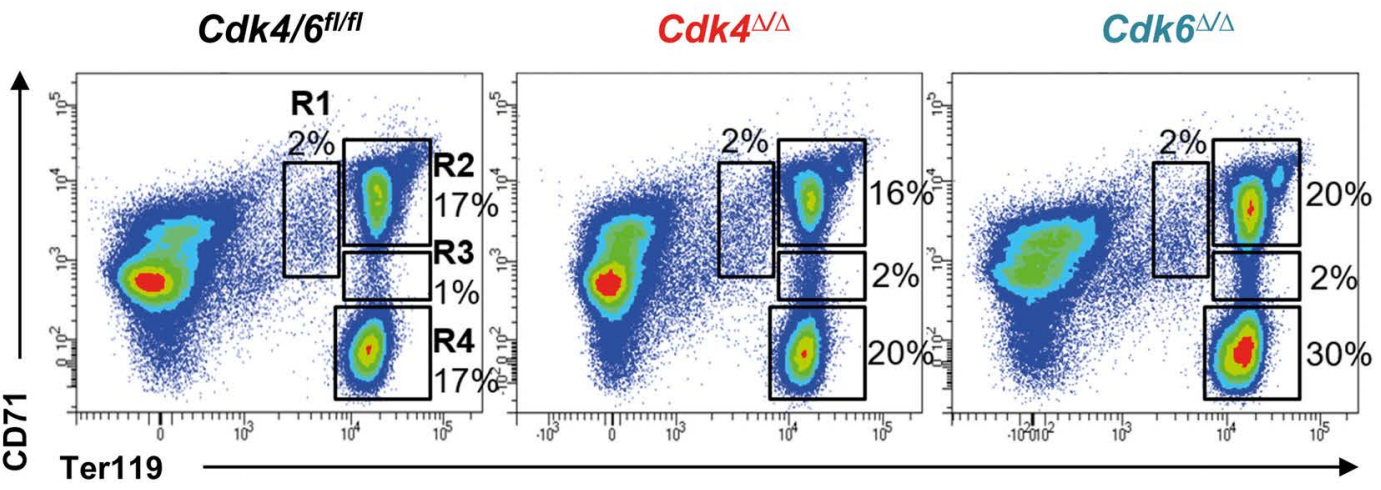

D erythroid development

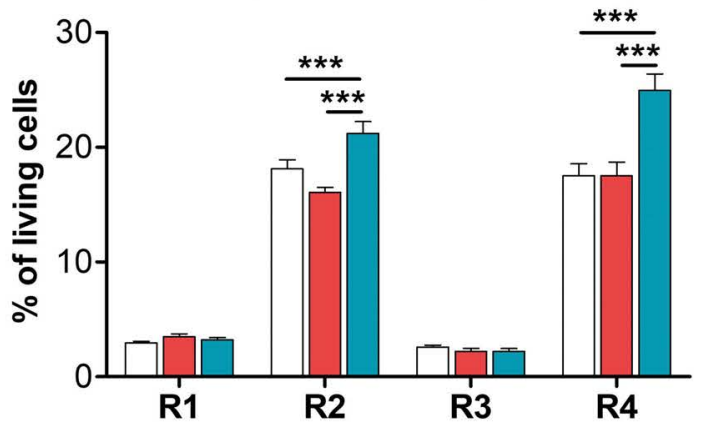

$C d k 4 / 6^{f l / f i}$
R2

R4

$C d k 4^{\Lambda / \Lambda}$
Figure 2. $C d k 6^{\Delta / \Delta}$ mice are anemic with compensatory upregulation of erythrocytes in the bone marrow. Analysis was performed 3 weeks post final polyinosinic-polycytidylic acid (poly(I:C)) injection. (A) Number of red blood cells (RBC) in peripheral blood ( $\mathrm{n} \geq 12$ for each group ( $\left.C d k 4^{\Delta / \Delta} ; C d k 6^{4 / \Delta} ; C d k 4 / 6^{n / / 1 /}\right)$. (B) Percentage of Ter $119^{+}$cells in bone marrow quantified by flow cytometry (Cdk4 $4^{\Delta / \Delta}$ : Cdk6 $6^{\Delta / \Delta}: C d k 4 / 6^{4 / 1 / 1}, n \geq 7 /$ genotype). (C) Gating strategy and representative flow cytometry pseudocolour plots for (D) quantification of erythroid development in the bone marrow (BM) based on CD71 and Ter119 expression from

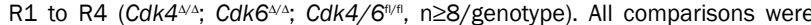
done with one-way ANOVA followed by Tukey's Multiple Comparison Test, $\star P \leq 0.05, * * P \leq 0.01, * * * P \leq 0.001, * * * * P \leq 0.0001$. 
harboring floxed Cdk4 or Cdk6 alleles and deleted CDK4 or CDK6 in adult hematopoiesis. We ascribed most hematopoietic side effects to the role of CDK6 in erythroid development, myeloid differentiation and hematopoietic stem cell activation.

Our results identify CDK6 inhibition as major determi- nant for anemia development. $C d k b^{\Delta \Delta}$ mice display low $\mathrm{RBC}$ counts, reduced hemoglobin and hematocrit levels. With the exception of an altered spleen size these findings mirror the situation in complete $\mathrm{Cdkb}^{-1}$ mice. ${ }^{6}$ Changes in erythroid cells are already present 3 weeks after CDK6 deletion. Cdk $4^{\Delta \Delta}$ mice show reduced RBC,

A
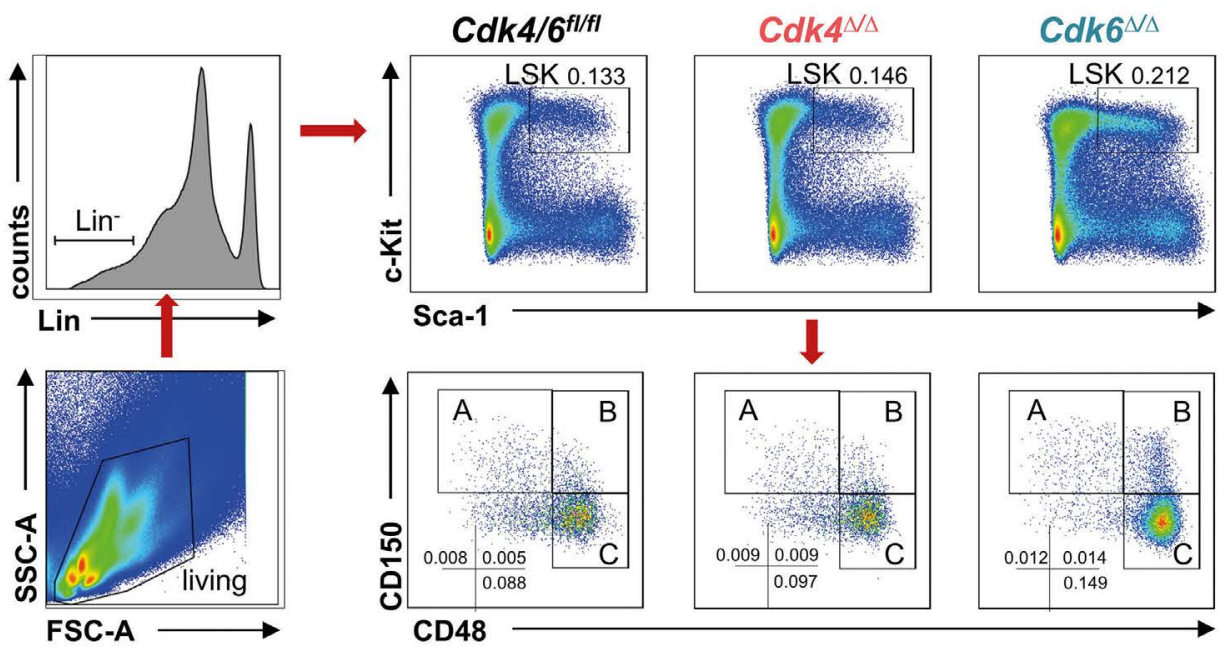

B

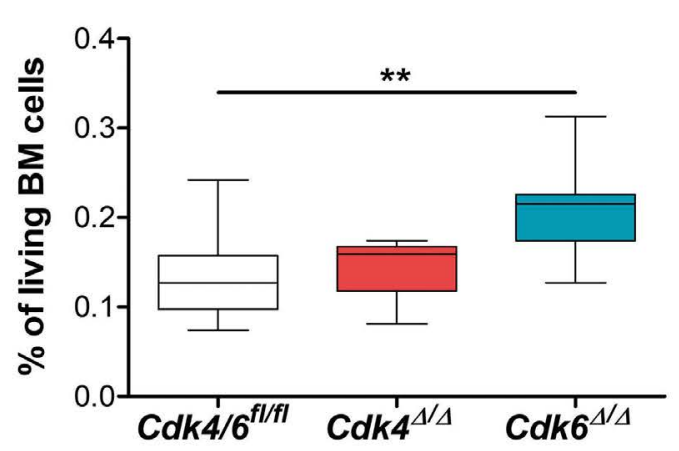

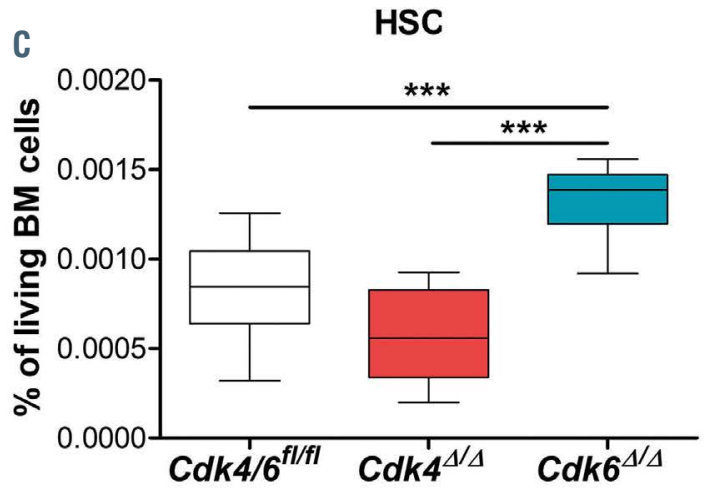

D

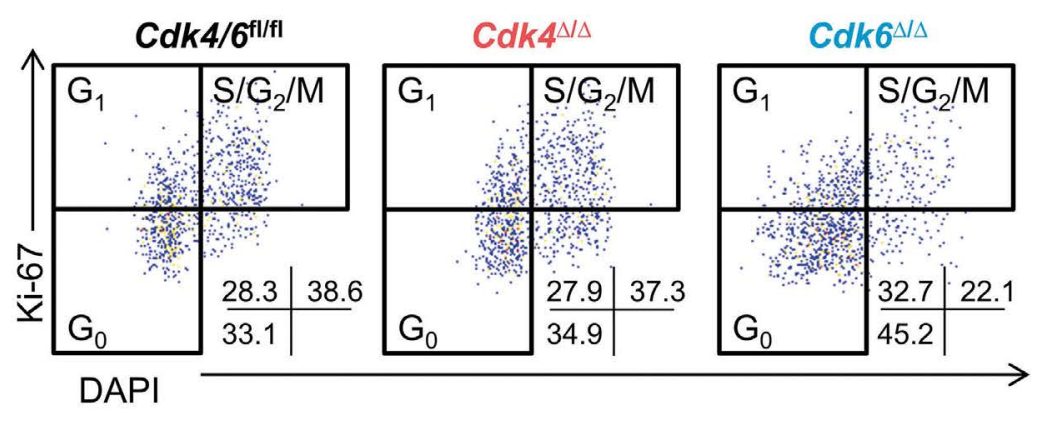

Fraction B 
while other blood parameters and erythroid development are unaltered, which may be explained by a slightly reduced cell cycle progression in reticulocytes in the absence of CDK4. Alterations in erythroid development are most likely linked to the additional functions of CDK6 beyond its role as a cell cycle kinase but as transcriptional regulator and contributor to the cytoskeleton of erythroid cells. ${ }^{6}$

CDK6 plays a minor role under homeostatic conditions for HSC maintenance in complete $C d k 6^{-1}$ mice where the most dormant HSC start to accumulate..$^{20}$ Upon deletion of CDK6 during adult hematopoiesis the numbers of all HSC fractions are elevated, which is not observed in the $\mathrm{BM}$ of $C d k 4^{\Delta / \Delta}$ mice. The global expansion of the stem/progenitor pool in $C d k 6^{\Delta \Delta}$ compared to $C d k 6^{-1}$ may reflect a key role for CDK6 at all HSC stages which is compensated for in the total knockout. The poly(I:C)-triggered interferon storm may also contribute to the differences as interferons induce HSC cycling. ${ }^{41,42}$ However, the time frames chosen make poly(I:C) effects unlikely as associated changes like increased Sca-1 expression resolve within 8 days. ${ }^{43} \mathrm{CDK} 6$, but not CDK4, has been implicated in stress-induced hematopoiesis. Murine C $d_{k} 6^{-1}$ cells only survive oncogenic stress by mutating the tumor suppressor Tp53 - a mechanism that has been confirmed in leukemia patients. ${ }^{17}$ The $C d k 6^{\mathrm{H} / \mathrm{l}}$ model now opens the opportunity of studying the consequences of CDK6 deletion in already established cancers circumventing Tp53 mutations.

Cdk4 mice have not been reported to exert hematopoietic abnormalities..$^{11,14}$ We found that CDK4 deletion in the murine adult hematopoietic system first triggers the accumulation of myeloid progenitors in the $\mathrm{BM}$ presumably contributing to the observed increase in BM cellularity, which declines at a later time point. These differences do not translate into different numbers of $\mathrm{CD} 11 \mathrm{~b}^{+} \mathrm{Gr} 1^{\mathrm{hi}}$ granulocytes in $C d k 4^{\Delta / \Delta}$ mice. Conversely, a significant reduction of $\mathrm{CD} 11 \mathrm{~b}^{+} \mathrm{Gr} 1^{\mathrm{hi}}$ granulocytes in $C d k 6^{\Delta \Delta}$ mice is seen, which probably induces a compensatory expansion of myeloid progenitors as observed 6 weeks after $C d k 6$ deletion. The increase in erythroid progenitors after 6 weeks supports the concept of a compensation taking place as response to the decreased numbers of mature erythroid cells. As aberrant type I interferon signaling accelerates myelopoiesis, ${ }^{44}$ the poly(I:C)induced interferon signaling might contribute to the initial abnormal expansion of myeloid progenitors in $C d k 4^{\Delta_{\Delta}}$ mice, while differentiation and maturation stay unaltered. During myeloid differentiation interleukin (IL)-6, IL-1 and IL-17 act on MPP and promote the production of myeloid cells in a feed-forward loop. ${ }^{45,46}$ CDK6 regulates cytokine expression in hematopoiesis ${ }^{18}$ and thus may
A

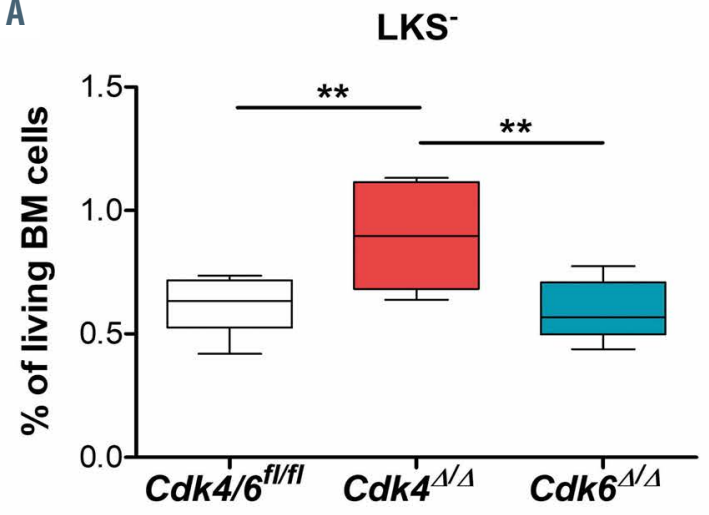

C

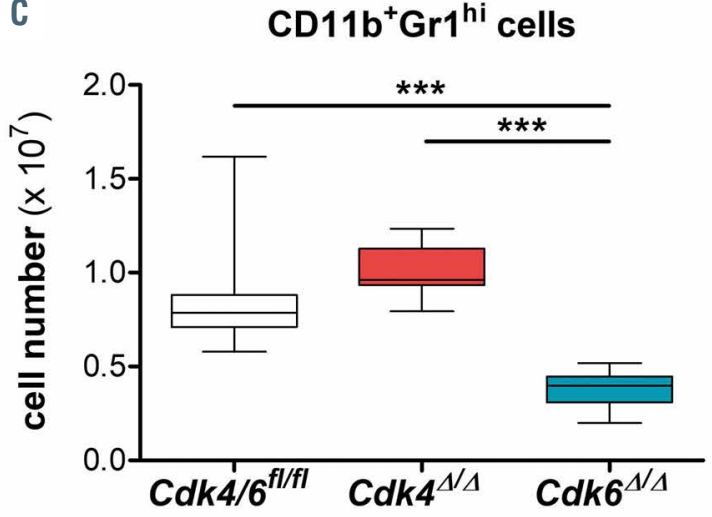

B

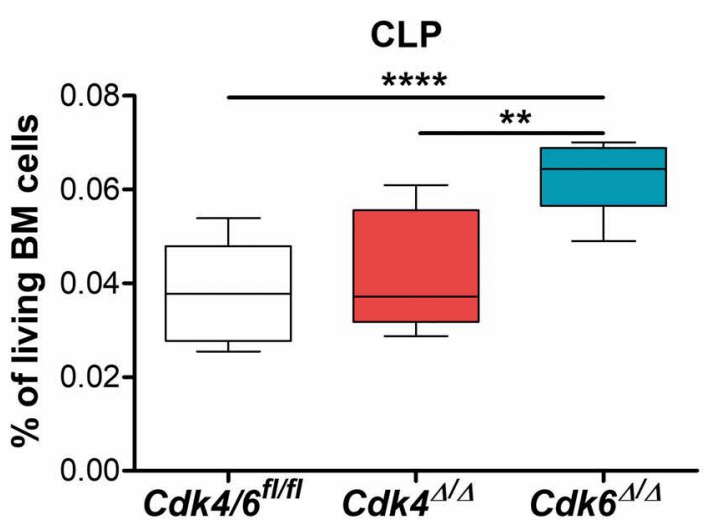

D

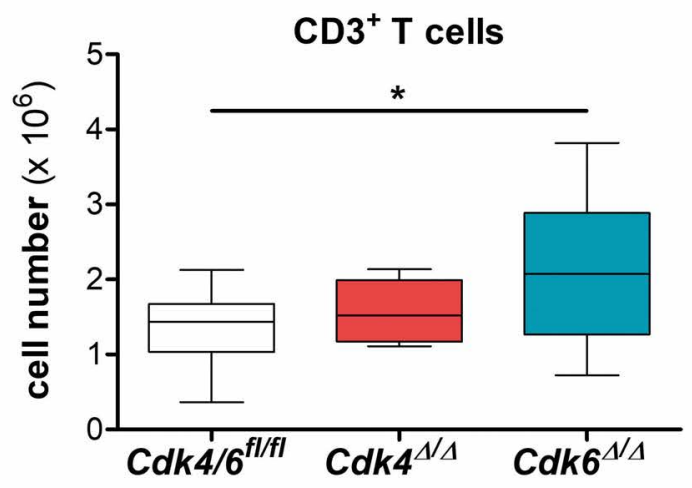

Figure 4. CDK4 and CDK6 drive the myeloid and lymphoid progenitor pool in opposite directions. Analysis was performed 3 weeks post final polyinosinic-polycytidylic acid (poly(I:C)) injection. Percentage of (A) myeloid progenitors (LKS-) and (B) common lymphoid progenitors (CLP) (LinIL-7R $\mathrm{R}^{+} \mathrm{C}-\mathrm{Kit}^{\mathrm{mid}} \mathrm{Sca}-\mathrm{1}^{\mathrm{mid}}$ ) per $2.5 \times 10^{6}$ bone

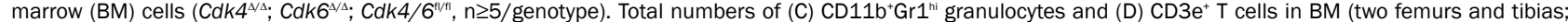
$C d k 4^{\Delta / \Delta} ; C d k 6^{\Delta / \Delta} ; C d k 4 / 6^{i / f l 1}, \mathrm{n} \geq 8 /$ genotype). All comparisons were done with one-way ANOVA followed by Bonferroni's Multiple Comparison Test, $* P \leq 0.05, * * P \leq 0.01$ $* * * P \leq 0.001, * * * * P \leq 0.0001$. 
contribute to the "control" of myeloid progenitor expansion. CDK4 deficiency may delocalize CDK6 from regulatory transcriptional complexes involved in cytokine production to its essential task in the cell cycle. A re-localization of CDK6 in the absence of CDK4 has been observed in melanoma, where CDK6 was "withdrawn" from its angiogenesis-promoting role. ${ }^{47}$

CDK6 interferes with myeloid differentiation by different ways: CDK6 inhibits granulocyte differentiation by blocking RUNX1 from interacting with $\mathrm{C} / \mathrm{EBP} \alpha$ and DNA binding. ${ }^{8} \mathrm{CDK} 6$, but not $\mathrm{CDK}$, inhibits myeloid differentiation in MLL-AF9 expressing leukemia ${ }^{9}$ and CDK6 deficiency ameliorates the hallmarks of JAK2 $2^{\mathrm{V} 1 \mathrm{17}}$-driven myeloproliferative neoplasms. ${ }^{18}$ In line, $C d k 6^{\Delta \Delta}$ mice display an impaired myeloid differentiation resulting in less $\mathrm{CD} 11 \mathrm{~b}^{+} \mathrm{Gr} 1^{\mathrm{hi}}$ cells mirroring the frequent occurrence of neutropenia in patients receiving CDK4/6 inhibitor treatment.

In $C d k 6^{-1}$ mice T-cell maturation is impaired.' We observed elevated numbers of CLP and $\mathrm{CD} 3^{+} \mathrm{T}$ cells in $\mathrm{BM}$ of $C d k 6^{\Delta \Delta}$ but not of $C d k 4^{\Delta \Delta}$ mice. CDK4/6 inhibitors affect T-cell subpopulations differentially: activation of effector $\mathrm{T}$ cells is enhance ${ }^{27,48}$ while regulatory $\mathrm{T}$ cells are suppressed. ${ }^{49}$ The accumulation of lymphoid progenitors pinpoints to an involvement of CDK6 in exiting the lymphoid progenitor state reminiscent of CDK6's role in leaving the quiescence state in HCS. Mx1-Cre induced gene deletion is inconsistent in the thymus (data not shown and ${ }^{50}$ ) impeding any T-cell development analysis.

Total $\mathrm{Cdk} 4^{-1}$ and $\mathrm{Cdk}^{-1}$ mice do not mirror the adverse events observed in CDK4/6 inhibitor-treated patients. The models presented in this study now enable the characterization of the specific roles of CDK4 or CDK6 in cell cycle and beyond as artefacts that appear in total knockout mice starting from embryogenesis are excluded. To date, only one tissue-specific study on the functions of CDK4 has been reported in adipocytes. ${ }^{51}$

CDK4- or CDK6-specific inhibitors would reduce side effects and represent a step towards precision medicine: while CDK4 inhibitors may be successful in hereditary melanoma where activating CDK4 mutations are frequent, ${ }^{5,53}$ acute myeloid leukemia is associated with aberrant CDK6 signaling. ${ }^{54}$ The inducible deletion of CDK6 represents an appropriate estimate for adverse effects of CDK6-targeting PROTACs. ${ }^{11-33}$

We here report the consequences of induced ablation of CDK4 and CDK6 in adult mice. The side-by-side analysis of induced deletion of CDK4 or CDK6 links anemia and neutropenia, both the most frequent adverse events in CDK4/6 therapy, to the inhibition of CDK6. The observed hematological side effects in patients may result from the accumulation of dormant HSC combined with defective myeloid differentiation and erythroid development after CDK6 inhibition.

Taken together, the novel $C d k 4^{\mathrm{At} / \mathrm{I}}$ and $C d k 6^{\mathrm{fH} / \mathrm{I}}$ mouse models are powerful tools to unravel cell type-specific mechanisms and side effects of CDK4/6 therapy to improve therapeutic treatment regimens.

\section{Disclosures}

No conflicts of interest to disclose.

\section{Contributions}

$B M, T B$ and MPM planned and conducted experiments and analyzed the data; SK did experiments; VS supervised the study; BM, TB, MPM and VS wrote the manuscript.

\section{Acknowledgments}

The authors would like to thank the Centre for Phenogenomics, Toronto, Canada, for generating Cdk4 and Cdk6 floxed mice. We are indebted to the animal caretaker as well as the genotyping team. Thanks to Philipp Jodl for excellent technical support.

\section{Funding}

This work has received funding from the European Research Council under the European Union's Horizon 2020 research and innovation program grant agreement 694354 (VS) and was also supported by the Austrian Science Fund grant SFB-F6107 and F6101.

\section{References}

1. Meyerson M, Enders GH, Wu CL, et al. A family of human cdc2-related protein kinases. EMBO J. 1992;11(8):2909-2917.

2. Tadesse S, Yu M, Kumarasiri M, Le BT, Wang S. Targeting CDK6 in cancer: state of the art and new insights. Cell Cycle. 2015;14(20):3220-3230.

3. Sherr CJ, Beach D, Shapiro GI. Targeting CDK4 and CDK6: from discovery to therapy. Cancer Discov. 2016;6(4):353-367

4. Bonelli M, La Monica S, Fumarola C, Alfieri R. Multiple effects of CDK4/6 inhibition in cancer: from cell cycle arrest to immunomodulation. Biochem Pharmacol. 2019;170:113676.

5. Malumbres M, Sotillo Ro, Santamaŕa D, et al. Mammalian cells cycle without the Dtype cyclin-dependent kinases Cdk4 and Cdk6. Cell. 2004;118(4):493-504

6. Uras IZ, Scheicher RM, Kollmann K, et al. Cdk6 contributes to cytoskeletal stability in erythroid cells. Haematologica. 2017;102(6): 995-1005.

7. Hu MG, Deshpande A, Schlichting N, et al. CDK6 kinase activity is required for thymo- cyte development. Blood. 2011;117(23): 6120-6131.

8. Fujimoto T, Anderson K, Jacobsen SEW, Nishikawa SI, Nerlov C. Cdk6 blocks myeloid differentiation by interfering with Runx1 DNA binding and Runx1C/EBPalpha interaction. EMBO J. 2007;26(9):2361-2370.

9. Placke T, Faber K, Nonami A, et al. Requirement for CDK6 in MLL-rearranged acute myeloid leukemia. Blood. 2014;124(1): 13-23.

10. Dickinson ME, Flenniken AM, Ji X, et al. High-throughput discovery of novel developmental phenotypes. Nature. 2016;537 (7621):508-514

11. International Mouse Phenotyping Consortium I. CDK4. 2020 [cited 2020 2020/04/12]; Available from: https://www.mousephenotype.org/data/ge nes/MGI:88357

12. Rane SG, Dubus P, Mettus RV, et al. Loss of Cdk4 expression causes insulin-deficient diabetes and Cdk4 activation results in $\beta$ islet cell hyperplasia. Nat Genet. 1999;22(1):44-52.

13. Tsutsui T, Hesabi B, Moons DS, et al. Targeted disruption of CDK4 delays cell cycle entry with enhanced p27(Kip1) activity. Mol Cell Biol. 1999;19(10):7011-7019.

14. Barrière C, Santamaría D, Cerqueira A, et al. Mice thrive without Cdk4 and Cdk2. Mol Oncol. 2007;1(1):72-83.

15. Jayapal SR, Wang CQ, Bisteau X, et al. Hematopoiesis specific loss of Cdk2 and $\mathrm{Cdk} 4$ results in increased erythrocyte size and delayed platelet recovery following stress. Haematologica. 2015;100(4):431-438.

16. Handschick K, Beuerlein K, Jurida L, et al. Cyclin-dependent kinase 6 Is a chromatinbound cofactor for NF- $\mathrm{BB}$-dependent gene expression. Mol Cell. 2014;53(2):193-208.

17. Bellutti F, Tigan A-S, Nebenfuehr S, et al CDK6 Antagonizes p53-induced responses during tumorigenesis. Cancer Discov. 2018;8(7):884-897.

18. Uras IZ, Maurer B, Nivarthi H, et al. Cdk6 coordinates Jak2V617F mutant MPN via NFKB and apoptotic networks. Blood. 2019;133(15):1677-1690.

19. Buss H, Handschick K, Jurrmann N, et al. Cyclin-dependent kinase 6 phosphorylates NF-KB P65 at serine 536 and contributes to the regulation of inflammatory gene expression. PLoS One. 2012;7(12):e51847.

20. Scheicher R, Hoelbl-Kovacic A, Bellutti F, et 
al. CDK6 as a key regulator of hematopoietic and leukemic stem cell activation. Blood. 2015;125(1):90-101.

21. Kollmann K, Heller G, Schneckenleithner C, et al. A kinase-independent function of CDK6 links the cell cycle to tumor angiogenesis. Cancer Cell. 2013;24(2):167-181.

22. Uras IZ, Walter GJ, Scheicher R, et al. Palbociclib treatment of FLT3-ITD+ AML cells uncovers a kinase-dependent transcriptional regulation of FLT3 and PIM1 by CDK6. Blood. 2016;127(23):2890-2902.

23. Hester A, Koenig A, Dobler F, et al. Palbociclib in daily clinical use: real world experience of the breast center at the University Hospital Munich. Ann Oncol. 2019;30(Suppl 3):iii58.

24. Sobhani N, D'Angelo A, Pittacolo M, et al. Updates on the CDK4/6 inhibitory strategy and combinations in breast cancer. Cells. 2019;8(4):321.

25. Schmoellerl J, Barbosa I, Eder T, et al. CDK6 is an essential direct target of NUP98-fusion proteins in acute myeloid leukemia. Blood. 2020;136(4):387-400.

26. Goel S, DeCristo MJ, McAllister SS, Zhao JJ. CDK4/6 inhibition in cancer: beyond cell cycle arrest. Trends Cell Biol. 2018;28(11): 911-925.

27. Deng J, Wang ES, Jenkins RW, et al. CDK4/6 inhibition augments antitumor immunity by enhancing T-cell activation. Cancer Discov. 2018;8(2):216-233.

28. European Medicines Agency E. Ibrance EPAR Product information. 2018 [cited 2020 2020/02/27]; Available from: https://www.ema.europa.eu/en/documents/product-information/ibrance-eparproduct-information_en.pdf

29. Chen X, Xu D, Li X, et al. Latest overview of the cyclin-dependent kinases $4 / 6$ inhibitors in breast cancer: the past, the present and the future. J Cancer. 2019;10(26):6608-6617.

30. Hu W, Sung T, Jessen BA, et al. Mechanistic investigation of bone marrow suppression associated with palbociclib and its differentiation from cytotoxic chemotherapies. Clin Cancer Res. 2016;22(8):2000-2008.

31. Brand M, Jiang B, Bauer S, et al. Homologselective degradation as a strategy to probe the function of CDK6 in AML. Cell Chem Biol. 2019;26(2):300-306.
32. De Dominici M, Porazzi P, Xiao Y, et al. Selective inhibition of Ph-positive ALL cell growth through kinase-dependent and independent effects by CDK6-specific PROTACs. Blood. 2020;135(18):1560-1573.

33. Rana S, Bendjennat M, Kour S, et al Selective degradation of CDK6 by a palbociclib based PROTAC. Bioorg Med Chem Lett. 2019;29(11):1375-1379.

34. Yang C, Li Z, Bhatt T, et al. Acquired CDK6 amplification promotes breast cancer resistance to CDK4/6 inhibitors and loss of ER signaling and dependence. Oncogene. 2017;36(16):2255-2264.

35. Li Z, Razavi P, Li Q, et al. Loss of the FAT1 tumor suppressor promotes resistance to CDK4/6 inhibitors via the Hippo pathway. Cancer Cell. 2018;34(6):893-905.

36. Bradley A, Anastassiadis K, Ayadi A, et al. The mammalian gene function resource: the International Knockout Mouse Consortium Mamm Genome. 2012;23(9-10):580-586.

37. Kuhn R, Schwenk F, Aguet M, Rajewsky K Inducible gene targeting in mice. Science. 1995;269(5229):1427.

38. Anampa J, Haque T, Murakhovskaya I, et al. Macrocytosis and dysplastic anemia is associated with the cyclin-dependent kinase 4/6 inhibitor palbociclib in metastatic breast cancer. Haematologica. 2018;103(3):e98e102.

39. Laurenti E, Frelin C, Xie S, et al. CDK6 levels regulate quiescence exit in human hematopoietic stem cells. Cell Stem Cell. 2015;16(3):302-313

40. Wilson A, Laurenti E, Oser G, et al. Hematopoietic stem cells reversibly switch from dormancy to self-renewal during homeostasis and repair. Cell. 2008;135 (6):1118-1129.

41. Frelin C, Herrington R, Janmohamed S, et al. GATA-3 regulates the self-renewal of longterm hematopoietic stem cells. Nat Immunol. 2013;14(10):1037-1044

42. Essers MAG, Offner S, Blanco-Bose WE, et al. IFN $\alpha$ activates dormant haematopoietic stem cells in vivo. Nature. 2009;458(7140): 904-908.

43. Velasco-Hernandez T, Säwén P, Bryder D, Cammenga J. Potential pitfalls of the Mx1 Cre system: implications for experimental modeling of normal and malignant hematopoiesis. Stem Cell Rep. 2016;7(1):11 18.

44. Buechler MB, Teal TH, Elkon KB, Hamerman JA. Cutting edge: type I IFN drives emergency myelopoiesis and peripheral myeloid expansion during chronic TLR7 signaling. J Immunol. 2013;190(3):886-891.

45. Reynaud D, Pietras E, Barry-Holson K, et al. IL-6 controls leukemic multipotent progenitor cell fate and contributes to chronic myelogenous leukemia development. Cancer Cell. 2011;20(5):661-673.

46. Schürch Christian M, Riether C, Ochsenbein Adrian F. Cytotoxic CD8+/+ T cells stimulate hematopoietic progenitors by promoting cytokine release from bone marrow mesenchymal stromal cells. Cell Stem Cell. 2014;14(4):460-472.

47. Kollmann K, Briand C, Bellutti F, et al. The interplay of CDK4 and CDK6 in melanoma. Oncotarget. 2019;10(14):1346-1359.

48. Schaer DA, Beckmann RP, Dempsey JA, et al. The CDK4/6 inhibitor Abemaciclib induces a $\mathrm{T}$ cell inflamed tumor microenvironment and enhances the efficacy of PD-L 1 checkpoint blockade. Cell Rep. 2018;22(11): 2978-2994.

49. Goel S, DeCristo MJ, Watt AC, et al CDK4/6 inhibition triggers anti-tumour immunity. Nature. 2017;548(7668):471475.

50. Kortylewski M, Kujawski M, Wang T, et al. Inhibiting Stat3 signaling in the hematopoietic system elicits multicomponent antitumor immunity. Nat Med. 2005;11(12):1314 1321.

51. Lagarrigue S, Lopez-Mejia IC, Denechaud P$\mathrm{D}$, et al. CDK4 is an essential insulin effector in adipocytes. J Clin Inves. 2016;126(1):335348

52. Meyle KD, Guldberg P. Genetic risk factors for melanoma. Hum Genet. 2009;126(4): 499-510.

53. Soura E, Eliades PJ, Shannon K, Stratigos AJ, Tsao H. Hereditary melanoma: update on syndromes and management. Genetics of familial atypical multiple mole melanoma syndrome. J Am Acad Dermatol. 2016;74(3): 395-410.

54. Uras IZ, Sexl V, Kollmann K. CDK6 inhibition: a novel approach in AML management. Int J Mol Sci. 2020;21(7):2528. 